\title{
La gestión del departamento de talento humano en la capacitación y desarrollo del profesorado ecuatoriano
}

\section{The management of the human talent department in the training and development of the Ecuadorian teaching staff}

Lic. Ericka Figueroa Martínez. Mgs

Dra. María Luisa Correa Reyes, Mgs.

Lic. Ana Gallegos Samaniego, Mgs.

Universidad de Guayaquil, Ecuador

Autor para correspondencia: ana.gallegoss@ug.edu.ec, ericka.figueroama@ug.edu.ec, ma_luisacorrea@hotmail.com

Fecha de recepción: 01 de Septiembre de 2016 - Fecha de aceptación: 01 de Noviembre de 2016

Resumen: Esta investigación trata sobre la reingeniería en el departamento de inspección general de las instituciones educativas con la finalidad de fortalecer y realizar cambios positivos para las instituciones de acorde a lo estipulado en nuestra ley educativa. La metodología de esta investigación fue un estudio de tipo bibliográfico. Se verifico y se comprobó las siguientes problemáticas existenciales: desvinculación del departamento de talento humano con la academia, la participación efectiva del docente en círculos de estudio (espacios generados en los horarios establecidos por el jefe de talento humano) y la falta de vínculos de redes colectivos de aprendizajes con la unidad educativa de manera nacional e internacional, es lo que se tratará en esta investigación. Existe un vacío en este tema debido a que revisando en las referencias bibliográficas las instituciones particulares en el Ecuador no apertura la movilidad para el perfeccionamiento docente. Los resultados más importantes fueron medibles y observables a través de las posturas científicas que demuestra el focus group donde se evidencia que el líder de esa muestra no gestiona la academia y ciencia dentro de su entorno educativo, es decir, no está cumpliendo su rol como autoridad que promueve la calidad de la institución educativa. La propuesta se basa en una línea operativa de los indicadores de talento humano.

Palabras claves: talento humano; capacitación; perfeccionamiento docente

Abstract: This research is about reengineering in the department of general inspection of educational institutions in order to strengthen and make positive changes for institutions according to what is stipulated in our educational law. The methodology of this research was a bibliographic study. The following existential problems were verified and verified: the disengagement of the human talent department with the academy, the effective participation of the teacher in study circles (spaces generated in the schedules established by the chief of human talent) and the lack of network links Collective learning with the educational unit nationally and internationally, is what will be discussed in this research. There is a vacuum in this subject because reviewing in the bibliographical references the particular institutions in Ecuador do not open the mobility for the improvement of teaching. The most important results were measurable and observable through the scientific positions shown by the focus group where it is evident that the leader of that sample does not manage the academy and science within its educational environment, that is, it is not fulfilling 
its role as an authority, which promotes quality in the educational institution. The proposal is based on an operational line of human talent indicators.

Key words: human talent; training; teacher training

\section{Introducción}

El presente proyecto tiene una constancia documentada sobre la necesidad imperiosa de reconocer las habilidades y destrezas que debe desarrollar la jefatura de inspección general en las instituciones educativas, puesto que se necesita revisar la bibliografía pertinente para darse cuenta que es necesario la activación de leyes que permiten fomentar la capacitación desde el departamento de inspección general.

Las consecuentes acciones del área de inspección en el área académica permiten que los docentes acrediten en su rol docente pues la investigación y la academia promueven una mejor clase con estrategias educativas inclusivas.

El rol del docente en el área de gestión permite que las actividades que realice sean avaladas por sus esfuerzos ya que su desempeño está en relación a la proyección académica de la misma, está generará una remuneración contemplativa de sus esfuerzos.

El objetivo del presente proyecto está en que la visión conceptual del inspector general está centrada en la operativización de sus funciones que es uno de los ejes de sus funciones pero la parte de capacitación como reza en los artículos de la ley no son claros y se diluyen en otras jefaturas.

\section{Desarrollo}

\section{Centros Educativos y elementos principales:}

El educador, crea un clima escolar impactante en tanto permita que su entorno educativo se realice con armonía y la comunicación sea asertiva de una manera organizada, pues este es el medio de estudio y compromiso en donde se generan los aprendizajes.

Las dependencias educativas que maneja el educador deben estar acorde con los respectivos cánones organizativos desde sus funciones claras, en un organizador gráfico para saber cuáles son sus deberes dentro de la gestión hasta la ergonomía de sus estadios de trabajo.

(Estepa, 2008) El proceso organizativo de una institución se aprovecha con una buena comunicación, pues este es el puntal del clima social de una organización pues permite manejar una infraestructura, equipo directivo, docente y discente con un hilo conductor llamado: educación.

El área de Talento Humano participa directamente en esta gestión pues operativamente relaciona la cantidad de estudiantes versus la cantidad docente y genera un cruce del personal el cual a través de horarios permite la organización de las asignaturas con el distributivo, inclusive direcciona al personal de servicio a la realización de sus labores. Crea un ambiente de armonía y cohesión propiciando con los expresos la salida y entrada de estudiantes pues la relación armónica 
es base fundamental, y esta esta evidenciada desde la planificación, evaluación y control de las diferentes jefaturas.

En el área de perfeccionamiento docente se crea enlaces académicos en las horas de Gestión Académica para la capacitación del personal docente .La entrada y salida (permisos) del personal docente debe ser verificada por el jefe de Talento Humano de esta manera se relaciona armónicamente el personal docente, discente y de servicio en pro de una institución proactiva.

\section{La economía es consecuente al elevado académico docente}

La participación del estudio continuo del departamento de Inspección General debe promoverse con la participación del Vicerrectorado Académico, puesto que el uno solicita las gestiones de interrelación de estudio presencial - distancia y la otra jefatura indica cuales son los temas relevantes que debe tomar en cuenta el profesorado según las nuevas tendencias.

(Velasquez, 2015) Los niveles de movilidad permiten al jefe de Talento Humano visualizar un ausentismo propio del perfeccionamiento docente, pero que es condescendiente con un plan de contingencia planificado y presentado en el POA (Proyecto Operativo Anual).

Las organizaciones mundiales como la UNESCO, BID, OEA son aquellos organismos que influyen directamente o indirectamente en las tendencias políticas de las naciones en cuestión de política educativa pública, por eso vemos reformas curriculares con tintes europeos por esto y más las tendencias del perfeccionamiento docente no es una moda es una manera de asegurar que la calidad del personal docente sea un referente de excelencia de la educación actual.

\section{La educación social y estado}

El perfeccionamiento docente, es parte de la política ecuatoriana pues los organismos de control de la educación promueven la inversión de fondos públicos en la educación como importante y fundamental eje de desarrollo de una nación.

(BID, 2013) En el CE latinoamericano la re contextualización de la visión macroeconómica produce relaciones (de continuidad entre niveles educativos, de interdependencia entre logros de desarrollo y logros educativos en educación

La formación del profesorado se encuentra como eje fundamental de la Formación Continua, pues es el mayor porcentaje de capacitaciones a nivel nacional esta direccionado a mejorar el perfil docente en las estrategias del proceso de enseñanza -aprendizaje, es rentable manejar este tipo de educación por la constante evaluación docente por parte del MINEDUC e INEVAL; inclusive los negocios particulares que ofertan de manera indirecta se ven beneficiado por este auge de exigencias del mejoramiento docente.

\section{Formación permanente del profesorado}


Como la educación es un asunto "integral" involucra lo social de una manera productiva, pues incluye el proceso formativo, pues es el saber de formación al capital humano es la base fundamental de cualquier política pública,

(Cebrian, 1997) Se maneja una relación intrínseca entre la preparación académica y el desarrollo del puesto de trabajo por eso las capacitaciones ayudan al docente a mejorar sus perspectivas de innovación y perfeccionamiento en áreas que desconoce porque un docente debe saber sobre la psicología aunque no es psicólogo, debe saber sobre economía pues si es directivo el tema presupuesto y compras públicas es determinante, aunque gestione otro departamento el conocimiento le dará al directivo-docente una visión real de su cosmogonía educativa. (Bolívar A. , 1997)

El desarrollo del currículo e innovaciones funciona mejor cuando, en lugar de ser dirigido por líderes o administración externa, se capacita a los propios profesores para tomar decisiones sobre cómo implementarlo.

El jefe de talento humano debe revisar la distribución de los horarios que está en congruencia con la cantidad establecida por el MINEDUC en horas dependiendo de las asignaturas designadas y que no es más que la búsqueda del perfil profesional en consecuencia de esto se ingresa un docente con la licenciatura adecuada y aquel que tenga mínimo una experticia de dos años.

(Antunez, 1998) Se debe propiciar planteamientos flexibles donde se distribuya un cronograma que permita reconocer no solamente un perfil de acuerdo a la formación permanente sino estrategias para incentivar con cursos y seminarios en el interior del país y exterior del país con recursos diferenciados, donde la principal herramienta será las redes institucionales.

Debería propiciarse desde dentro del propio centro, a través de planteamientos flexibles y de una distribución de los recursos conveniente. También a través de la ayuda externa mediante recursos y estímulos adecuados y una formación permanente ofrecida en condiciones favorables para el profesorado: horarios, lugares,... Otras estrategias para facilitar y apoyar el cambio consisten en fomentar el trabajo en equipo: planificar e impartir clases en común; elaborar, seleccionar materiales y evaluar en común; intercambiar experiencias entre seminarios y equipos de profesores $\mathrm{y}$ entre centros diferentes.

De ahí radica la importancia del Departamento de Talento Humano en el perfeccionamiento docente, capacitaciones internas y externas, certificar las horas extra curriculares con incentivos económicos y promover a su personal al ascenso.

\section{Liderazgo Transformacional}

El liderazgo del departamento de talento humano en una institución educativa, es aquel que engloba la academia y la investigación; por eso la búsqueda del perfeccionamiento docente, es importante desde sus propios propósitos, apoyo - seguimiento al docente y la cultura desde sus conocimientos hacia la proyección de nuevos conocimientos. 
(Bolívar, 1997)El "liderazgo transformacional" como modelo en la reestructuración escolar, recoge las dimensiones significativas utilizadas en otras conceptualizaciones, es decir, se promueve un liderazgo que permite el dialogo entre los docentes y autoridades con el fin de llegar acuerdos y propuestas en común.

El liderazgo compartido, es importante porque los monitores educativos podrían ser el personal seleccionado para ser los que replican a sus compañeros, utilizando las dos horas establecidas por el gobierno nacional para que sus compañeros puedan utilizarlas en ese requerido perfeccionamiento docente.

\section{Características del Liderazgo Educativo}

Posturas del líder educativo (Talento Humano)

1. Liderazgo y posición formal ocupada en la organización.

2. Liderazgo y gestión son diferentes

3. El liderazgo es inevitablemente político

4. El liderazgo es inherentemente simbólico

5. El liderazgo tiene algo que ver con un conjunto de cualidades humanas y éticas.

La educación en el Ecuador está cambiando y le está dando más valor al conocimiento como los escribió Francis Baccon "El conocimiento es poder" debido a que la educación es la que le brindará una mayor visión al docente de los cambios vanguardistas generacionales.

(Educación, 2010) Del desarrollo profesional.- El desarrollo profesional de los y las docentes del sistema educativo particular conduce al mejoramiento de sus conocimientos, habilidades, competencias y capacidades que les permitirán ofrecer un mejor servicio educativo. Los y las docentes de las instituciones educativas particulares podrán participar en los procesos de formación continua ofrecidos por la Autoridad Educativa Nacional.

Las propuestas que indica el Ministerio de Educación a través de su Programa de Fortalecimiento a Docentes Fiscales enlaza el conocimiento generalista de: Proyectos Colaborativos y Autónomos, Didáctica General - Especial y además con un perfeccionamiento en Técnicas Activas.

El docente ecuatoriano tiene diferentes proyecciones con el Modelo Pedagógico Ecológico ya que le permite integrar en su formación docente las diferentes asignaturas con la visión cosmogónica de la educación integradora que involucra: la familia, su entorno escolar, su medio social hasta incluso el impacto de las redes sociales en su entorno personal y educativo.

\section{Talento Humano en la Educación Ecuatoriana}

Ley Orgánica de Educación Intercultura

Art. 46.- Inspección General

Las funciones de Inspección General están contemplada en los albores 
1. Coordinar a los inspectores de grado o curso

2. Registrar la asistencia y puntualidad de docente y estudiantes

3. Gestionar el clima organizacional y promover y controlar el cumplimiento de las normas de convivencia y de la disciplina en el plantel;

4. Publicar los horarios de clases y exámenes

5. Organizar la presentación del estudiantado en actos sociales, culturales, deportivos y de otra índole.

6. Conceder el permiso de salida de las estudiantes para ausentarse del plantel durante la jornada educativa.

7. Aprobar la justificación de dos a siete días consecutivos

8. Llevar los siguientes registros: archivo documental de cada docente, distributivo de trabajo docente, horarios de clase, asistencia de las estudiantes, solicitudes de justificación por inasistencia a clases de los estudiantes debidamente firmada por su representante legal.

9. Asegurar el cumplimiento de los estándares de calidad educativos emitidos por el Nivel Central de la Autoridad Educativa Nacional.

10. Los demás que delegue el Rector o Director.

Entre las que delegue el Rector en el siguiente proyecto indicamos departamento de Talento Humano, como se lo denomina en la actualidad, responda a los cánones de esta jefatura incluyendo el área de Formación y Perfeccionamiento Docente.

Las funciones del área de talento humano en el aspecto academia a través del alcance del manual de procedimiento determinarán las acciones de perfeccionamiento docente tanto en el ámbito nacional e internacional.

Entre los indicadores que debe tener un gestor de talento humano o inspector general dentro de un área educativa son:

\begin{tabular}{|c|c|}
\hline Indicadores & Centro Innovador \\
\hline $\begin{array}{l}\text { Estructura } \\
\text { Atmósfera }\end{array}$ & $\begin{array}{l}\text { Flexible, mucho uso de grupos de trabajos temporales. Fácil cambio en las líneas de actuación y } \\
\text { en las tradiciones. Múltiples vinculados basados en la colaboración funcional. Controles } \\
\text { definidos de forma Amplia. Territorio móvil. } \\
\text { Centrada en la persona, calidad y calidez }\end{array}$ \\
\hline $\begin{array}{l}\text { Filosofía y } \\
\text { Actitudes } \\
\text { de Liderazgo }\end{array}$ & $\begin{array}{l}\text { Cohesión } \\
\text { Liderazgo } \\
\text { Motivador laboral y personal }\end{array}$ \\
\hline Toma decisiones & $\begin{array}{l}\text { Direcciona Operativamente dentro de la institución educativa } \\
\text { Promueve la academia } \\
\text { Promueve e perfeccionamiento docente }\end{array}$ \\
\hline $\begin{array}{l}\text { Mediación de } \\
\text { Conflictos }\end{array}$ & $\begin{array}{l}\text { Coordina espacios armónicos del Buen Vivir ( Código de Convivencia) } \\
\text { - Autoridades } \\
\text { - Docentes } \\
\text { - Estudiantes } \\
\text { - Padres de Familia y Representantes Legales } \\
\text { - Comunidad }\end{array}$ \\
\hline
\end{tabular}




\section{El Educador Nacional}

La educación nacional promueve el perfeccionamiento docente tanto en el área particular como fiscal, siendo la formación continua una manera de acercamiento docente en las áreas de enseñanza con los programas que el MINEDUC imparte o de manera personal realiza cada docente para su mejoramiento.

\section{Materiales y Métodos}

- Diseño: Cualitativo y Bibliográfico

- Variables: Talento Humano en Centros Educativos y Perfeccionamiento Docente.

- Campos de acción: Educativo

- Sujetos: Instituciones Particulares

\section{Resultados}

Los resultados de la investigación bibliográfica son significativas ya que se mide el nivel de congruencia que existe entre el perfeccionamiento docente y los niveles de compromiso del líder de talento humano en relación del rendimiento de las destrezas docentes, pues el trabajo del profesorado mide el grado de satisfacción de los estudiantes tanto en rendimiento académico y niveles de investigación (docente-estudiante).

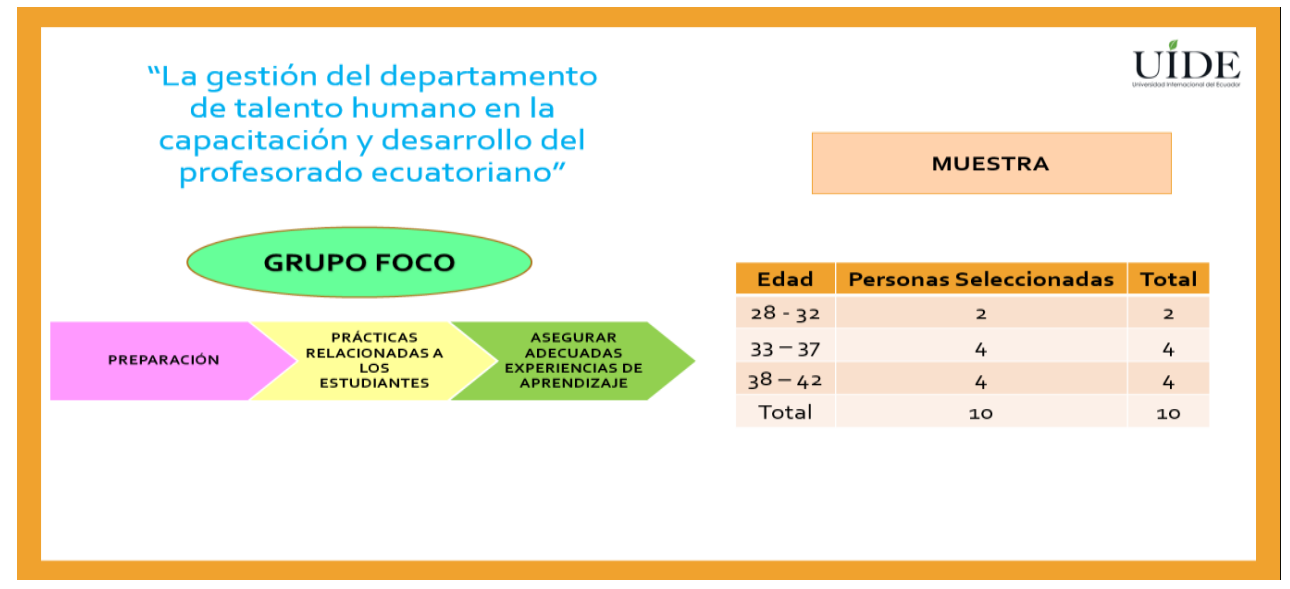

La brecha consignada del ente nominador y las personas que administran en la operativización de los reglamentos de la LOEI (Jefes de Talento Humano) es el resultado que arroja este Focus Group y la solución se centra en la activación el perfeccionamiento docente no solo como un derecho sino como una obligación y estas acciones deben estar encaminadas a la movilidad, plan de contingencia y gestión del conocimiento.

\section{Discusión}

Los objetivos del estudio de gestión de la autoridad nominadora (talento humano) han sido develados con el argumento bibliográfico que cumple la normativa de instituciones nacionales e 
internacionales, el grado de importancia de este estudio radica en que desarrollar acuerdos y enlaces con colectivos educativos, es la manera correcta de interrelacionar y vincular a través de acuerdos a los docentes con el perfeccionamiento docente, el mismo que debe ser el que busca la forma de mediar entre la academia y esa pertinencia de contenidos relevantes para dichas capacitaciones.

Entre las debilidades de este estudio se encuentra la falta de documentos actualizados que traten del tema siendo esto una causa por la cual es necesario trabajar y desarrollar esta ponencia.

\section{Conclusiones}

El impacto funcional de esta investigación es que los entes rectores de la educación nacional verán en este documento una base teórica y práctica a desarrollar en su estándar de gestión.

El impacto social de esta investigación está direccionado a los docentes que ven en su desarrollo profesional un desafío que algunas veces no pueden lograr pero con el direccionamiento del jefe de talento humano institucional se pueden llegar acuerdos mediáticos con centros educativos nacionales o internacionales.

El impacto de originalidad del presente proyecto está basado en una guía de procedimientos del departamento de inspección o talento humano que le confiera el espacio logístico y académico pertinente a esta jefatura.

\section{Bibliografía}

Antunez Sánchez. (2012). Curso online Redacción Científica y las Tecnologías de la Información las Comunicaciones. Obtenido de www.veterinaria.org/revistas/redvet/n080806.html

Antunez, S. (1998). Claves para la organizacion de centros escolares. Editorial Horsori.

BID. (2013). Latinoamerica.

Bolívar. (1997). Liderazgo,mejora y centros educativos.

Bolívar, A. (1997). Liderazgo, mejora y centros educativos.

Cebrian, M. (1997). Nuevas competencias para la formalidad inicial y permanente del profesorado.

Curso de Redacción. (2005). Obtenido de http://www.calamoycran.com/curso_detalle.php?

Cursos gratis.[página web en la Internet]. emagister. (2015). Obtenido de http://www.emagister.com/cursos-gratis/curso-gratisredaccion-kwes-759.htm

Day, R. (1996). ¿Cómo escribir y publicar trabajos científicos?. . Washington DC: Organización Panamericana de la. 
Educación, R. d. (2010).

Estepa, P. M. (2008). Las percepciones del clima escolar por directivos,docentes y alumnado mediante el empleo de "redes semántica naturales".Su importancia en la gestión de los centros educativoa. (350 Septiembre -Diciembre).

Estrada Cuzcano, A. (2006). Curso Redacción científica y publicaciones académicas. Facultad de Letras y Ciencias. Obtenido de http://redaccioncientifica.blogspot.com/

Mari Mutt, J. (S/f). Manual de redacción científica. Obtenido de http://www.caribjsci.org/

Ramirez Padrón. (2007). Bases para la redacción del escrito científico. Curso preevento Pedagogía 2007. Obtenido de http:/www.ispcmw.rimed.cu/sitios/

Velasquez, M. I. (2015). La educación en el discurso pedagógico oficial i un asunto educativo? VI(17). 